\title{
Antwort auf v. Platos vier Thesen zur Sozialfaschismus-Theorie
}

\section{Niels Kadritzke}

Die Replik v. Platos auf meine Kritik an seinem Neuaufguß der Sozialfaschismus-Theorie ist insofern entwaffnend, als sie sich in wesentlichen Punkten gar nicht auf meine Position bezieht, weil v. Plato diese entweder entstellt wiedergegeben oder gar nicht verstanden hat.

Das versetzt mich in die Lage, anstelle einer ausführlichen Gegenkritik auf meine ursprüngliche Polemik in PROKLA Nr. 11/12 zurückzuverweisen und mich an dieser Stelle mit einer Antwort zu begrügen, die in aller Kürze auf v. Platos vier Thesen eingeht und erneut aufzeigt, daß der Sozialfaschismus-These ein weitgehendes Unverständnis für die materiellen Grundlagen sowohl des Reformismus als auch des Faschismus zugrundeliegt.

Vorweg will ich festhalten, daß v. Plato auf die meisten jener für seine Beweisführung wichtigen Punkte nicht eingeht, in denen ich ihm unwissenschaftliches Vorgehen nachgewiesen habe. Auf diese Kritik einzugehen, hätte erfordert, die reklamierten Fehler entweder zu bestreiten oder aber sie einzugestehen, um sodann ihre Tragweite mithilfe anderer, als durchschlagender ausgewiesener Belege anstelle der nachweislich schiefen Interpretationen, falschen Zitate und trüben Quellen zu relativieren. Stattdessen gibt v. Plato den Vorwurf unwissenschaftlicher Fälschungen an mich zurück. Soweit sich dieser Vorwurf mit dem ersten Blick auf meine angeblichen ,Fälschungen und Unterstellungen" von selbst erledigt (1), brauche ich

1 So habe ich (vgl. Anm. 1 bei v. Plato) nicht behauptet,

- v. Plato gehe auf Trotzkis konkrete Einheitsforderungen überhaupt nicht ein, sondern vielmehr bemängelt, daß er sie im Zusammenhang mit den Auswirkungen der Sozialfaschismus-Propaganda auf die Einheitsfrontpolitik nicht bexücksichtigt und damit Trotzkis diesbezügliche Kritik an der KPD um eine wichtige Dimension verkürzt (PROKLA 11/12, S. 61, Anm. 9).

- v. Plato habe die zwei von mir hervorgehobenen unterschiedlichen Begründungen der Sozialfaschismus-These gar nicht gesehen, sondern vielmehr kritisiert, daß er sich über die Vieldeutigkeit des Begriffes in der Verwendung durch die KPD als Problem ganz allgemein hinwegsetzt und in seinen eigenen Definitionen einen fest umrissenen Sinn des historischen Begriffs simuliert (PROKLA 11/12, S.63).

- v. Plato sei auf das „Programm zur nationalen und sozialen Befreiung..." nicht ausführlich genug eingegangen, vielmehr habe ich seine unkritische Einschätzung dieses Programms bemängelt (PROKLA 11/12, S. 61, Anm. 11; den an dieser Stelle plazierten allzu spitzfindigen Seitenhieb gegen den KSV ziehe ich zur̈ick, weil in der Tat offenbleiben muß, ob es sich bei der Verkehrung der Reihenfolge von ,national" und ,sozial" in der KSV-Publikation um eine bewußte Fälschung oder um eine unbewußte Kritik an der KPD von 1930 gehandelt hat).

Schließlich habe ich lediglich konstatiert, daß v. Plato auf S. 175 seines Buches explizit die Lohntheorien der Nöltings mit der Braunthals gleichsetzt, obwohl die angeführten Zitate einander direkt entgegenstehen. Ich habe auf diesen für jedermann ersichtlichen Widerspruch nur beiläufig hingewiesen (PROKLA 11/12, S. 68) und keinerlei „Verwi- 
mich dazu nicht ausfuhrlich zu äußern. Interessant bleibt deshalb nur ein Punkt, in dem v. Plato meine Kritik richtig wiedergibt, ohne allerdings rechtzubehalten: unser Autor besteht auf der "faschistischen" Ablehnung der Frauenarbeit durch den ADGB und zeigt damit seine Unfähigkeit, ganz offensichtliche Irrtumer zu korrigieren. Der Wille zum Nachweis der vorausgesetzten These bringt vielmehr neue Fehlschlüsse hervor; denn natürlich wird das Einkommen einer Arbeiterfamilie im Durchschnitt erhöht, wenn die Männer die Arbeitsplätze der Frauen einnehmen und damit das einzige Familieneinkommen in eine höhere Lohnkategorie ruickt (2).

Wer den Unterschied zwischen dieser reformistischen Forderung des ADGB und der faschistischen Ideologie von der ,naturrlichen Bestimmung" der Frau als Hüterin von Haus, Herd und möglichst blonden Kindern nicht sieht (3), der will eben keinen Unterschied zwischen Reformismus und Faschismus wahrhaben. Auch in seinen Antworten auf die mir zugeschriebenen Thesen demonstriert v. Plato, wie Reformismus und Faschismus ununterscheidbar ineinander verschwimmen, wenn man das politische Panorama der Weimarer Zeit von einem Standpunkt aus beschreibt, der - hoch über den wirklichen Verhältnissen angesiedelt -- weder Klassenbasis noch politische Funktion von Reformismus und Faschismus, und schon gar nicht ihre jeweilige Beziehung zu den konkreten Interessen des Kapitals genauer zu betrachten erlaubt.

So versucht v. Plato in seinen Ausführungen zu These II für die Zeit vor 1933 den aktiven Willen der ADGB-Führung nachzuweisen, die reformistischen Gewerkschaften in ,faschistische Gewerkschaften" umzuwandeln. Er stuitzt sich dabei auf einen überinterpretierten Protokollauszug, der natürlich über die wirkliche politische Taktik des ADGB gegenüber den politischen Bemühungen des Generals v. Schleicher, den linken Flügel der NSDAP und die freien Gewerkschaften für seine Pläne einer ,,sozialen“" Militärdiktatur zu gewinnen, keine authentische Auskunft geben kann (4). Dagegen zeigt die Ablehnung einer gewerkschaftlichen Beteiligung an der Querfront Schleichers im Dezember 1932, daß sich innerhalb von SPD und ADGB die skeptische Haltung gegenüber einer von der Reichswehr getragenen Lösung durchgesetzt hat, die im übrigen - was wohl auch v. Plato nicht behaupten

schungsideologie" daraus gemacht. Die prinzipiellen theoretischen Unklarheiten v. Platos habe ich im Gegenteil gerade an den grundä̈tzlichen Fragestellungen zu demonstrieren gesucht.

2 Wobei nicht zu vergessen ist, daß die ADGB-Forderung sich auf einen Zustand bezieht, in dem schon die Bestimmungen der Arbeitslosenversicherung dahin wirkten, daß die Arbeiterfamilien in der Regel nur Einkommen aus einer Quelle bezogen haben. Genau diese Voraussetzung läßt v. Plato offensichtlich außer Acht, wenn er behauptet (Anm.1), die Durchsetzung der ADGB-Forderung würde die Familieneinkommen verringert haben.

3 An diesem faschistischen Ideologem gibt es im übrigen eine präzise und ausführliche Kritik des ADGB. Vgl. J. Grünfeld, Frauenarbeit und Faschismus, in: Die Arbeit, 9. Jg. 1932, S. 424 ff.

4 Eine authentische Interpretation der ADGB-Absichten müßte zusätzlich zu den Protokollen externer Sondierungsgespräche die Auf́zeichnungen ADGB-interner Beratungen zu diesem Thema heranziehen. Solche unumgänglichen quellenkritischen Ủberlegungen sind der urwüchsigen historischen Methode v. Platos aber konsequenter- und bezeichnenderweise fremd geblieben. 
will - keineswegs mit der faschistischen Diktatur selbst identisch gewesen wäre.

Für die Zeit nach der "Machtergreifung" habe ich demgegenüber die Überlegungen skizziert, die der ADGB-Führung ihre Eingliederung in die NS-Diktatur akzeptabel und möglich erscheinen ließen. Diese Kapitulationsbereitschaft ändert aber nichts an der objektiven Tatsache, daß die Gewerkschaften durch die NS-Diktatur zerschlagen wurden. Diesen Vorgang kann v. Plato weder wahmehmen noch klassenpolitisch einschätzen, weil er ganz offensichtlich von der späteren Existenz. „faschistischer Gewerkschaften" im Sinne „ständischer" Arbeiterorganisationen ausgeht, welche sich im Rahmen ,faschistischer Arbeisgemeinschaften" artikulieren konnten. Er demonstriert damit aber nur seine vollständige Unkenntnis des wirklichen Gesichts der NS-Diktatur, die entgegen ihrer eigenen Ideologie niemals distinkte Organisationen von Arbeitern zulassen konnte, sondern die Arbeiter in der „Deutschen Arbeitsfront" als einer „Eirheitsorganisation von Einzelarbeitgebern und Einzelarbeitnehmern ${ }^{6}$ (F. Neumann) wie in den einzelnen „Betriebsgemeinschaften" dem kapitalistischen Führer-Eigentümer unterstellt und damit ihre Klassenqualität viel offener enthïllt hat, als es ihre korporativistische Ideologie verrät (5).

Vollends konfus wird v. Plato in seiner Antwort auf These IIl, die mir eine ziemlich unsinnige Behauptung unterstellt. Denn ich habe gerade gegen seine Interpretation darauf bestehen müssen, daß die reformistischen Theorien der Zwischenkriegszeit den demokratischen Staat der bürgerlichen Gesellschaft als Übergangsform zum Sozialismus auffassen und sich genau darin qualitativ von allen faschistischen Staatstheorien unterscheiden. Demgegenüber unterstellt v. Plato den reformistischen Theorien eine faschistische Qualität, weil sie angeblich die Möglichkeit eines ,,Sozialismus innerhalb des Kapitalismus“" ideologisierten.

Dabei ist es gerade eine der Vorbedingungen für die Wirkung des Weimarer Reformismus in der Arbeiterklasse, daß die SPD im Gegensatz etwa zu ihrer Godesberger Programmatik von heute am sozialistischen Endziel festhielt, was mit der gleichermaßen reformistischen, von v. Plato betonten Anschauung durchaus vereinbar war, durch sukzessive staatliche Eingriffe ließen sich sozialistische Teilmomente schon vor der schließlichen Umwälzung der Produktionsverhältnisse in der kapitalistischen Gesellschaft verankern.

Ich habe nun lediglich behauptet, diese optimistische Auffassung vom unaufhaltsamen linearen Hineinwachsen in den Sozialismus sei durch die Krise erheblich gestört worden (6), ohne daß die SPD programmatisch auf die Vergesellschaftung der Produktionsmittel als Voraussetzung für den vollendeten Sozialismus verzichtet hätte. Diese Tatsache entzieht aber seiner These von der faschistischen Qualität der sozialdemokratischen Theorie-Entwicklung den Boden, weil alle Varianten faschisti-

5 Jede einigermaßen saubere bürgerliche Sozialgeschichte der NS-Diktatur sieht in diesem Punkt klarer als v. Plato, von den vorliegenden marxistischen Analysen ganz zu schweigen. Vgl. H. G. Schumann: Nationalsozialismus und Gewerkschaftsbewegung, Hannover und Frankfurt a. M. 1958.

6 Dies läßt sich mannigfach belegen, u. a. durch das Referat Tarnows auf dem Leipziger Parteitag von 1931, in dem Hilferdings wirtschaftsdemokratischer Optimismus von 1927 deutlich revidiert wird. 
scher Ideologie den Staat bekanntlich als Garanten einer gesellschaftlichen Harmonie bei grundsätzlich unveränderten Eigentums- und Produktionsverhältnissen vorstellen.

In seiner Entgegnung auf These IV versucht v. Plato schließlich, im Nachhinein jene Konfusion ubersichtlich zu machen, die er in seinem Buch mit der Theorie der Wirtschaftsdemokratie und der Politik der Arbeitsgemeinschaften angerichtet hat.

Uns kann dabei relativ gleichgültig bleiben, ob er -- in vollendet idealistischer Wahrnehmung - eine in der konjunkturellen Stabilitätsphase begründete Theorie während der Krise in eine theoretisch ganz anders begründete Praxis ,umschlagen“ zu lassen beliebt, anstatt materialistisch zu analysieren, wie die wirtschaftsdemokratische Ideologie unter dem Druck der ökonomischen Krise auch von den überzeugtesten Reformisten zurückgestellt werden muß.

Wichtig ist vielmehr die Darstellung der angeblichen Arbeitsgemeinschaftspolitik selbst. V. Plato behauptet in seinem Buch, daß 1930 eine erneute Zentrale Arbeitsgemeinschaft installiert wurde, in der bis 1933 die lohn- und sozialpolitischen Klassenkompromisse ausgehandelt wurden. Dieser Begriff beschreibt eine regelmäßige Institution, die als zentrale Klärungs- und Beschlußinstanz für eine staatsfreie und dennoch umfassend verbindliche Regelung der zentralen Probleme im Verhältnis von Kapital und Arbeit zu sorgen hat. Eine solche Institution hat es nach 1930 micht gegeben und ihre Existenz wird von unserem Autor auch nicht mehr behauptet (7).

Stattdessen entschließt er sich jetzt, die Formen der Konfliktregulierung, die in der ganzer: Weimarer Zeit rechtlich festgelegt und praktiziert wurden, als Arbeitsgemeinschaft zu bezeichnen. Das kann man machen, wenn man jede rechtlich normierte Auseinandersetzung zwischen Kapital und Arbeit im Rahmen der bürgerlichen Gesellschaft schon als Arbeitsgemeinschaft bezeichnen will. Ich kann darin keinen Sinn erkennen, weil der Begriff sehr wohl dazu taugt, auf den verschiedenen Ebenen der Klassenauseinandersetzungen Phasen des quasi friedlich ermittelten Interessenausgleichs von Phasen des verschärften Verteilungskampfes zu unterscheiden, wobei beide Formen dex Klassenbeziehungen Möglichkeiten reformistischer

Vgl. die Schilderung der gescheiterten Verhandlungen, auf deren bloße Ankündigung v. Plato seine These gestützt hat, in: Die Angestellten-Bewegung 1928-1931 (hrsg. vom AfA-Bund) Berlin, 1931, S. 71 f. Diese Verhandlungen haben aus gewerkschafticher Sicht ganz eindeutig erwiesen, ,daß die Unternehmer als Voraussetzung für die Gesundung der wirtschaftlichen Verhältnisse, eine Umkehr auf dem Gebiete der Finanz-, Wirtschafts- und Sozialpolitik forderten, die letzten Endes der freigewerkschaftlichen Zielsetzung geradezu entgegengesếzt war . . . Die Politik der Arbeitgeber und ihrer Organisationen hat in der Krisis zu einer Verschärfung des sozialen Kampfes geführt, die eine Verständigung durch solche wohlgemeinten Verhandlungen mit ihren Spitzenorganisationen unmöglich machte" (ebd., S. 72). Vgl. auch U. Hüllbusch: Die deutschen Gewerkschaften in der Weltwirtschaftskrise, in: W. Conze/H. Raupach (Hrsg.), Die Staats- und Wirtschaftskrise des Deutschen Reiches 1929/33, Stuttgart 1967, S. 146 f. Ich habe die Tatsache der Beteiligung des ADGB an diesen Sondierungsgesprächen zunächst ubersehen, ihr Ergebnis bestätigt aber vollständig meine These von der ökonomischen Unmöglichkeit einer erneuerten Arbeitsgemeinschaft (vgl. PROKI A 11/12, S. 75 f.). 
Politik darstellen, die allerdings auf unterschiedlichen politischen und ökonomischen Rahmenbedingungen beruhen ( 8 ).

Daß spätestens seit dem Ende der Großen Koalition - und schon angekündigt durch den Nordwest-Eisen-Streit 1928 - der verschärfte Verteilungskampf arbeitsgemeinschaftliche Konfliktlösungen unmöglich gemacht hat, kann nur leugnen, wer den defensiven Charakter der reformistischen Konfliktstrategie - angezeigt durch die von der Arbeitslosigkeit beeinflußte Streikscheu der Gewerkschaften (9) und ihre Konzentration auf Reallohnbehauptung $b z w$. staatliche Zwangsschlichtung zur Begrenzung von Lohnsenkungen usw. - als Indiz für konsensuale Klassenzusammenarbeit mißversteht. Dies ist bei v. Plato zweifellos der Fall, weil er die politischen Ziele und Perspektiven der Kapitalseite vollständig außer acht läßt, die seit 1928 in sich ständig verschärfender Polemik gegen den ,Tarifstaat" im allgemeinen und gegen Schiedsurteile der Schlichtungsstellen im besonderen zu Felde zog. Denn diese haben in ihren Augen aufgrund der Mitwirkung reformistischer Funktionäre jene in der Krise erforderlichen Lohnsenkungen verhindert, welche die Kapitalseite im offenen Schlagabtausch mit den durch ảie Furcht vor der zunehmenden Arbeitslosigkeit gelähmten einzelnen Abteilungen der Arbeiterklasse durchzusetzen entschlossen war (10). V. Platos völlig äußerliche, auf die Resultate der staatlichen Zwangsschlichtung fixierte Betrachtungsweise kann gar nicht herausfinden, in welchem Maße selbst die reformistische Defensivstrategie, die lediglich auf eine Begrenzung der materiellen Einbußen für die Arbeiterklasse gerichtet war, im Verlaufe der ökonomischen Krise mit der Klassenpolitik des Kapitals unvereinbar geworden ist.

Damit ist schon der grundlegende Mangel angesprochen, der in w. Platos Haupthese iiber den Charakter der Sozialdemokratie zum Ausdruck kommi und daruberhinaus seiner Kritik an meiner angeblichen These I zugnundeliegt. Meiner Charakterisierung der sozialỏemokratischen Politik als reformistisch setzt er die Feststellung entgegen, die SPD-Führung habe seit 1914 ,im Wesen eine Politik des Imperialismus" betrieben. Abgesehen von der unscharfen Formulierung ist an dieser Entgegensetzung außerordentlich erstaunlich, daß

1. sich für v. Plato die reformistische und die in der Tat prinzipiell konterrevolutionäre Funktion der Sozialdemokratie gegeneinander ausschließen, und

2. unser Autor den Reformismus nur dort am Werk sieht, wo wirklich positive Reformen passieren. Wo solche Rếormen zugunsten der Arbeiterklasse nicht mög. lich sind, wie in der Weltwirtschaftskrise, kann es demzufolge auch keinen Reformismus geben. Ahnlich wie bei der verharmlosenden Beschreibung faschistischer Politik bringt v. Plato auch hier ideologische Versprechungen und realisierbare Lei-

8 Diese Unterschiede bedurfen zweifellos auch in der aktuelen gewerkschaftspolitischen Entwicklung einer genaueren Darstellung und Anaiyse.

9 Dieser Zusammenhang ist ein Thema für sich. Hier genügt der Hinweis, daßo die Thesc, die Streikfahigkeit der Arbeiterklasse setze einen bestimmten Beschäftigungsgrad voraus, keinesfalls schon mit den Hinweis auf ihre apologetische Funktion im reformistischen Denken erledigt ist.

10 Für diese Agitationsrichtung, die das sozialpolitische Leitmotiv für die faschistische Orientierung insbes. der Schwerindustrie angibt, gibt es eine Fülle von Belegen. Vgl. insbes, die offrzjelien Entschließungen des RdI und anderer Kapitalvertretungen. 
stungen begrifflich zur Deckung; jedoch hat ein solch unmittelbarer Begriff von Reformismus nicht nur mit dem von mir verwendeten Begriff nichts zu tun, sondern ist auch so unsinnig wie die Beschränkung des Begriffs Sozialist auf bewußte Mitglieder sozialistischer Gesellschaften.

Indem v. Plato uns seinen unsinnigen Reformismus-Begriff unterschiebt und diesen sodann für unvereinbar erklärt mit der Erkenntnis der prinzipiell konterrevolutionären Rolle des Reformismus, erschleicht er sich für seine Sozialfaschismus-These den alleinigen Anspruch, sowohl die Erscheinung eines „Reformismus ohne Reformen" als auch die konterrevolutionäre Rolle der Sozialdemokratie analysieren zu können.

Daß der Reformismus in seiner praktischen Krisenpolitik die Arbeiterinteressen letztilich dem Rückgang der kapitalistischen Konjunktur ausliefert, ist als $\mathbb{Z}$ wangsgesetz reformistischer Politik in der Krise völlig unumstritten und steht im Zentrum nicht nur der historischen, sondern auch der aktuellen Reformismus-Kritik., wie sie in dieser Zeitschrift formuliert wird (11).

Die Beteiligung des Reformismus am Abbau reformistischer Errungenschaften bewegte sich aber seit 1929 in einem Widerspruch, den y. Plato in keiner Weise begriffen hat: den Erfordernissen der Krisenbewältigung kann die reformistische Politik mit Rücksicht auf ihre Klassenbasis gerade nicht in dem Maße Rechnung tragen, das die Kapitalseite für erforderlich hält und mit zunehmend antidemokratischen Mitteln durchzusetzen versucht. V. Platos Sündenregister der Müller-Regierung beschreibt also lediglich die eine Seite des widersprüchlichen Versuchs der Großen Koalition, die Krise wirtschaftspolitisch zu steuern.

In dieser Darstellung, über deren schlampig ermittelte Einzelheiten in diesem Zusammenhang hinweggesehen sei, kommen zwei ebenso schwerwiegende wie charakteristische analytische Fehler zum Vorschein. Zum einen beschreibt er die ,Verantwortung ${ }^{\prime 6}$ der Sozialdemokratie für die ,ungeheure Verelendung " der Arbeiterklasse bis 1930 wie ein bürgerlicher Oppositionsstratege, welcher die jeweils ,verantwortliche" Regierung für alle gesellschaftlichen Entwicklungen einer Legislativperiode staatspolitisch haftbar machen will; oder, am Beispiel verdeutlicht: wie der populistische Demagoge F. J. Strauß, der die sozialliberale Koalition von heute für die aktuelle Inflationsrate ,verantwortlich" macht, die bekanntlich eher etwas mit ökonomischen Erscheinungen auf Weltmarktebene zu tun hat. Zum anderen aber unterschlägt v. Plato - indem er sich auf die nackten Resultate der sozialpolitischen Exekution des ökonomischen Krisenverlaufs durch einen bürgerlichen Staat beschränkt, der sich noch nicht seines reformistischen Kostgängers entledigt hat - die weit über dessen reformistisch gebremstes Programm hinausschießenden Forderungen und langfristigen Perspektiven des Kapitals. Diese zielen auf eine unmittelbare Verschärfung des lohn- und sozialpolitischen Abbaus und wollen darüber hinaus langfristig verhindern, daß mit dem Konjunkturaufschwung die aktiven reformistischen Bestrebungen zur lohn- und sozialpolitischen Kompensation der Arbeiterklas-

11 So auch der Aufsatz über die ,Bedingungen sozialistischer Gewerkschaftsarbeit" in PROKLA Nr. 13 und der über die ,"Theorie“" vo Oertzens von Heimann/Zeuner in diesem Heft. 
se die Verwertungsbedingungen des Kapitals erneut begrenzen. Indem er diese Stoßrichtung der faschistischen Diktatur gegen die reformistische Politik iberhaupt nicht sieht, kann v. Plato die sukzessive Verdrängung der Sozialdemokratie nicht als Moment des Faschisierungsprozesses ausmachen, damit aber auch die klassenpolitische Tendenz dieses Prozesses in keiner Weise interpretieren (12).

Nur wer, wie v. Plato, die Politik der SPD ohne Bezug auf die klassenpolitischen Bestrebungen der Kapitalseite darstellt, kann die bürgerliche Krisenpolitik der Großen Koalition bis 1930 ihrem Inhait nach als sozialfaschistische Diktatur charakterisieren, wie es v. Plato jetzt in schneidiger Zuspitzung aller schon in seinem Buch angesammelten Fehlurteile ganz offen tut. An dieser These vom, sozialfaschistischen" Inhalt der gerade an der kapitalgerechten Bewältigung der ökonomischen Krise gescheiterten bürgerlich-reformistischen Koalition zeigt sich ganz deutlich, zu welch blamablen Resultaten eine vergleichsweise aufwendige ,materialistische ${ }^{6 \text { Un- }}$ tersuchung kommt, wenn sie die ökonomischen und politischen Erfolgsbedingungen und Existenzvoraussetzungen sowohl des Reformismus als auch des Faschismus nicht auf dem Boden der bürgerlichen Gesellischaft und aus den Krisen ihrer ökonomischen Reproduktion zu verstehen versucht.

Daß damit nicht einmal die jeweils spezifische Funktion von Reformismus und Faschismus für die bürgerliche Gesellschaft verstanden ist, zeigt sich hinsichtlich des Faschismus an den aufgezeigten Unklarheiten über das Verhältnis faschistischer Diktaturen zu jeder Art von Gewerkschaften ebenso wie in der Annahme, der reale Faschismus repräsentiere politisch, was er ideologisch verspricht, nämlich die „Arbeisgemeinschaft in der Krise“. Sein Unverständnis für Voraussetzungen und Funktion des Reformismus spiegelt sich darin, daß v. Plato auf zwei Fragen keine

12 Vor der Frage, warum der Gewehr bei Fuß stehende Sozialfaschismus zur Krisenlösung nicht herangezogen wurde, muß v. Plato entsprechend kapitulieren. Außer einem historisch völlig unergiebigen Hinweis auf die ganz anders gelagerten polnischen Verhältnisse, hält er lediglich auf der subjektiven Ebene einen angeblichen Boweis für den Willen der SPD-Führung zur sozialfaschistischen Diktatur bereit. Die Rede von O. Wels, die im übrigen in die Zeit vor dem manifesten Beginn der Weltwirtschaftskrise fällt, ist allerdings seinerzeit von den Kapitalvertretern nicht als großzügiges sozialfaschistisches Angebot, sondern als erneuerte Drohung einer sozialdemokratischen Diktatur des Proletariats verstanden und entsprechend kommentiert worden. Natürlich ist eine solche Interpretation so demagogisch wie die v. Platos, denn bei dieser großmäuligen Reklamation des Rechtes auf ,diktatorischen Republikschutz" handelt es sich lediglich um die illusionäre Vorstellung, die SPD könne in ihrem Zustand von 1929 - anders als noch 1923 - für eine klassenneutrale Überwindung politischer Krisen geradestehen, weil sie, wie Wels meinte, als einzige Partei ,die Garantie für eine Rückkehr zur Demokratie" biete, wenn die Krise überwunden sei (vgl. S. 14 des Protokolls: Sozialdemokratischer Parteitag Magdeburg 1929, Berlin 1929).

Die von Braun u. a. ausgedrückten Hoffnungen auf ein alsbaldiges Abwirtschaften einer an die Macht gelangten NSDAP, die v. Plato als weiteren Beweis für die sozialfaschistische Einstellung der SPD-Führung anführt, demonstrieren alles andere als die Bereitschaft, sich an einer solchen Diktatur zu beteiligen. Im übrigen stellt die Herausstellung solcher Zitate eine skrupellose Geschichtsfälschung dar, wenn nicht zugleich darauf hingewiesen wird, daß die KPD im selben Zeitraum ebenfalls dieser fur die Arbeiterbewegung äußerst verhängnisvollen Abwirtschaftungs-These anhing, die sich lediglich mit einer unmittelbaren Revolutionserwartung verknüpfte. 
Antwort weiß, die für das Verhältnis von Reformismus und Faschismus entscheidend sind:

1. Warum die SPD im Verlaufe der Krise aus allen Positionen verdrängt wurde, die ihr die wirtschaftspolitischen Entscheidungen des bürgerlichen Staates zu beeinflussen erlaubten.

2. Warum die wirklichen Faschisten nach 1933 auch die reformistischen Organisationen restlos zerschlagen und ihre ,sozialfaschistischen " Repräsentanten konsequent verfolgt haben (13).

Im Begriff des Sozialfaschismus fallen also die Unfähigkeit zu einer historisch-materialistischen Analyse des Reformismus wie des Faschismus zusammen, weil beider politische Funktion für die Aufrechterhaltung der kapitalistischen Produktionsweise sozusagen konjunkturneutral gleichgesetzt wird, anstatt ihr Verhältnis zueinander im Kontext der Veränderungen bürgerlicher Klassenpolitik unier dem Druck der ökonomischen Bewegung zu analysieren.

Das zeigt sich nirgends deutlicher als in der nunmehr auch offiziellen Programmformulierung der selbsternannten ,Partei der Arbeiterklasse ${ }^{6 \varepsilon}$, die - wie zu erwarten war - die Sozialfaschismus-Propaganda auf die heutige Sozialdemokratie anzuwenden versucht. Wenn es hier heißt, die ,Progromhetze gegen fortschrittliche Kräfte $^{66}$ und der, ,vorbeugende Terror ${ }^{66}$ der SPD-Führung mache diese zum geeigneten "Vollstrecker einer nochmaligen faschistischen Entwicklung", so läuft dies auf nichts anderes hinaus als auf die in v. Platos Buch noch vorsichtig kritisierte radikale Sozialfaschismus-Version $\mathrm{P}$. Merkers, die den Reformismus in Ländern mit stark entwickelter Arbeiterklasse als adäquaten Exekutor der faschistischen Diktatur vorsieht (14).

Angesichts dieser programmatischen Aussage enledigt sich jener mildernde Umstand, den v. Plato in seiner Replik geltend macht, für die Einschätzung seiner Sekte von selbst: die jetzige Politik der SPD-Regierung sei noch nicht als sozialfaschistisch bezeichnet worden. Denn daß die Politik der SPD - nach einer frappierenden Zirkeldefinition im Programmentwurf (15) - erst nach Errichtung der sozialfaschistischen Diktatur auch wirklich sozialfaschistisch ist, kann nicht darüber hinwegtäuschen, daß v. Platos famose „Partei des Proletariats" die SPD-Führung von heute ebenso wie die H. Muiller-Regierung von 1930 für eine Verkörperung des Sozial faschismus hält.

Abschließend zwei Bemerkungen zu v. Platos melodramatischem Appell, ,den Kommunisten" - und das meint nicht nur pauschal die Weimarer KPD, sondern jeden, der sich heute für einen Kommunisten ausgibt - mehr Glauben zu schenken als den reformistischen Ideologen, die SPD schärfer zu kritisieren als die KPD usw.

13 Auf meine Kritik an v. Platos absolut lächerlicher Antwort auf diese Frage in seinem Buch (vgl. PROKLA 11/12, S. 84) geht der Autor in seiner Replik bezeichnenderweise nicht ein.

14 Vgi. PROKLA 11/12, S. 64

15 Danach hängt die Frage, ob die Monopolbourgeoisie sich der SPD für ihre faschistischen Zwecke bedient, davon ab, ,ob die Sozialdemokratie durch den Einbau des reaktionären Gewerkschaftsapparates in das System staatlicher Unterdrückung eine sozialfaschistische Diktatur errichten kann" (S. 21 des Programmentwurfs). 
Abgesehen davon, daß die damit implizierten Vorhaltungen meine Position nicht betreffen, liegt solcher Gesinnungs-Methodologie die Unterstellung zugrunde, wer die Sozialfaschismus-These als ein theoretisches Produkt der revolutionären Partei der Arbeiterklasse von gestern und heute ablehne, verharmlose damit die politische Funktion der Sozialdemokratie und rechtfertige gar ihre Politik vor der Arbeiterklasse.

Zum einen kann es bei der historischen Analyse einer schwerwiegenden Niederlage der Arbeiterklasse nicht darum gehen, ,den Kommunisten ${ }^{66}$ mehr oder weniger Glauben $z u$ schenken, sondern nur darum, ihre politischen einschließlich der theoretischen Fehler in aller Schärfe zu analysieren, soweit sie zu einer solchen Niederlage beigetragen haben. Das Gejammer über die prinzipielle Gemeinheit des Reformismus und seinen doch viel größeren Anteil an der Niederlage kann dabei die KPD zuallerletzt entlasten, denn: Nur wo der Reformismus in der Vertretung von Arbeiterinteressen notwendig versagt, hat die revolutionäre Arbeiterbewegung eine historische Chance gehabt, den politischen Zielen der Arbeiterklasse insgesamt einen Schritt näherzukommen. Wo arbeiterfeindliche reformistische Politik nicht zu einem Moment erfolgreicher sozialistischer Politik geworden ist, hat zuallererst die revolutionäre Partei der Arbeiterklasse versagt. Und wo die reformistische Politik dazu herhalten muß, die Niederlage der Arbeiterbewegung als unausweichlich auszuweisen, liegt in der Regel ein Versuch vor, die Fehler der revolutionären Richtung zu bemänteln und die Analyse dieser Fehler zu behindern.

Zum anderen muß uns die moralisierende Grundtendenz in v. Platos Argumentation zu denken geben. Jede moralisierende Darstellung des Reformismus verfehlt seine wirkliche klassenpolitische Funktion und läuft auf eine idealistische Distanzierung von den tatsächlichen Verhältnissen in der Arbeiterschaft hinaus, weil sie den Reformismus nicht als Zustand von Arbeiterbewußtsein unter dem Eindruck bestimmter Erscheinungen des kapitalistischen Reproduktionszusammenhangs analysiert, und damit auch nicht die materiellen Bedingungen für den politischen Prozeß seiner Überwindung begreifen kann (16).

Nicht diejenigen also verharmlosen den Reformismus, die die materiellen Grundlagen seiner ideologischen Wirkung in der Arbeiterklasse jeweils dem Entwicklungsgang der kapitalistischen Ökonomie entsprechend zu bestimmen versuchen, sondern umgekehrt: diejenigen unterschätzen theoretisch und verniedlichen politisch die Bedeutung des Reformismus, die die Sozialdemokratie als sozialfaschistische Karrikatur propagieren und den Arbeitern mit diesem monströsen Popanz reformistisches Bewußtsein auszutreiben versuchen.

Eine lediglich moralisierende Kritik am Reformismus und das lamentierende Selbstmitleid des „Revolutionärs ${ }^{66}$ ergänzen sich in der Argumentation v. Platos gegenseitig und repräsentieren das klassische Syndrom sektiererischer Hilflosigkeit vor den wirklichen Problemen der Arbeiterklasse.

Der richtigen Forderung sozialistischer Arbeiterpolitik, an die der bürgerli-

16 Der Vorwurf, eine Weißwaschung des blutbefleckten Reformismus zu betreiben, beherrscht auch die ansonsten fast nur mit Entstellungen meiner Kritik arbeitende Polemik gegen meinen Aufsatz in der KSV-Zeitung, ,Dem Volke dienen“ (Mai 1974). 
chen Gesellschaft verhafteten Momente im Arbeiterbewußtsein anzuknüpfen, kommt die politische Programmatik studentischer Sekten nur in der hoffnungslos verdrehten Form nach, daß sie mit einer idealistisch begründeten Mission auf das Proletariat losgeht, um dessen Bewußtsein vermittels moralisierender Gegen-Propaganda einfach umzudrehen.

Einen in letzter Wirkung harmloseren Gegner als einen, der die reformistische Wirksamkeit des Reformismus derart eklatant unterschätzt, kann sich die Sozialdemokratie von heute gar nicht wünschen.

17 Vgl. dazu Rabehl/Spohn/Wolter: Historische und politischelVoraussetzungen der Kritik Mandelbaums am Sozialdemokratismus und Leninismus, Nachbemerkung zu: K. Mandelbaum, Sozialdemokratie und Leninismus, Zwei Aufsätze, Berlin 1974, S. 104 f. Zum Verhältnis reformistischen Arbeiterbewußtseins zur Oberfläche der kapitalistischen Reproduktion vgl. insbes. die einschlägigen Darlegungen in den Arbeiten des Projekts Klassenanalyse.

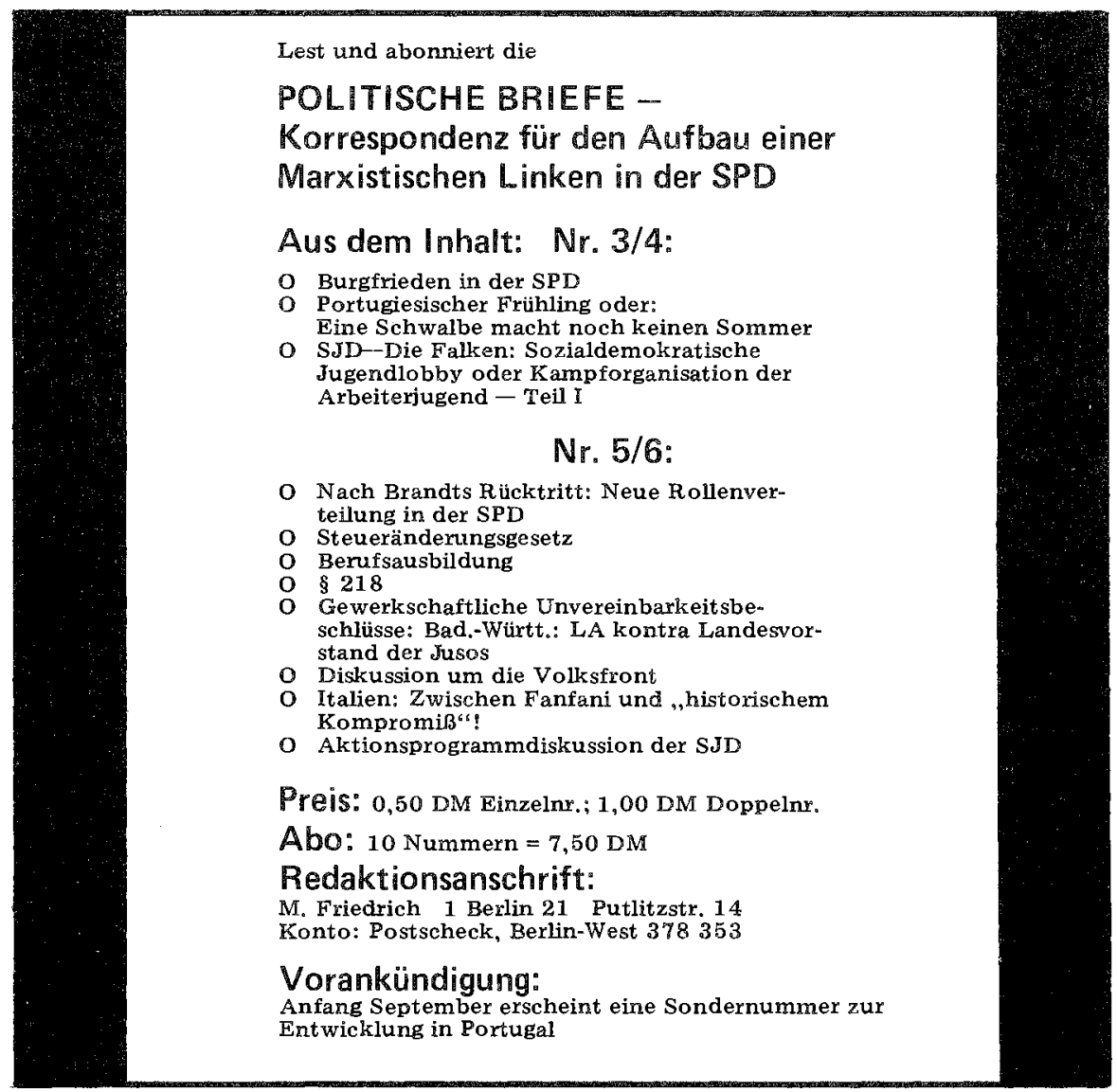

